\title{
VARIETIES OF GROUPS
}

\author{
BY B. H. NEUMANN ${ }^{1}$
}

1. Introduction. This lecture should really be given by Hanna Neumann, because her book with the same title is going to be published very shortly by Springer-Verlag, in the series "Ergebnisse der Mathematik"; and she gave an expository address with the same title at the Group Theory Conference at Canberra in August, 1965, and this also will be published very soon by Gordon and Breach in the Proceedings of that conference. All I can do is to follow the advice of that well-known junior mathematician Tom Lehrer and plagiarize.

2. Varieties. "Varieties" are equationally defined classes of algebras: starting from a "species" of algebras, that is the class of algebras with given numbers of algebraic operations of given kinds, unary, binary, and so on, we single out subclasses by imposing certain "laws" or "identical relations". Thus, for example, a single binary operation defines the species of "groupoids"; if the associative law is imposed on the operation, we obtain the variety of "semigroups". The study of varieties was initiated by Garrett Birkhoff in 1935; the name "variety" was introduced by Philip Hall in 1949. Much of the theory belongs to Universal Algebra, but I shall be mainly concerned with varieties of groups, because that is where the deepest theorems have been proved and the most difficult and intriguing problems remain open. To mention some examples of varieties of groups and to fix some notation:

$\mathfrak{D}$ is the variety of all groups;

(F is the variety of trivial (=one-element) groups;

$\mathfrak{A}$ is the variety of abelian groups, singled out by the commutative law $x y=y x$;

$\mathfrak{B}_{e}$ is the "Burnside" variety of groups of exponent $e$, singled out by the law $x^{e}=1$;

$\mathfrak{A}_{e}=\mathfrak{B}_{e} \cap \mathfrak{A}$ is the variety of abelian groups of exponent $e$.

This last example incidentally illustrates the fact that an intersection of varieties is again a variety; not only an intersection of two

${ }^{1}$ This is an amplified version of an address delivered on January 27,1967 , at the Annual Meeting, in Houston, by invitation of the Committee to Select Hour Speakers for Annual and Summer Meetings; received by the editors March, 1967. 
varieties but of arbitrarily many. It follows that the varieties in a species, or the subvarieties of a variety -in our case the subvarieties of $\mathfrak{N}$, that is, the varieties of groups-form a complete lattice under the "contained in" order relation. What can be said about this lattice? It is modular but not distributive. How big is it? Here we come at once to the first major unsolved problem.

Problem 1. How many varieties of groups are there?

All we know is that the cardinal number of the set of group varieties lies between $\boldsymbol{\aleph}_{0}$ and $2 \boldsymbol{\aleph}_{0}$. The same question can be asked for other algebraic systems; for each of the sets of varieties of groupoids, of quasi-groups, and of loops the answer is $2 \aleph_{0}$ [5].

Next we may ask about chain conditions in the lattice of group varieties. One easily shows that the ascending chain condition is not satisfied, for example, by considering

$$
\mathfrak{A}_{2} \subset \mathfrak{A}_{4} \subset \mathfrak{A}_{8} \subset \cdots \subset \mathfrak{A} \text {. }
$$

Problem 2. Does the lattice of varieties of groups satisfy the descending chain condition?

The two problems are not unrelated: if the answer to the second were to be "yes", then the answer to the first would have to be " $\boldsymbol{\aleph}_{0}$ " - but I guess that in both cases the answer goes the other way. We shall meet Problem 2 later in a different form.

A classical theorem of Garrett Birkhoff gives an alternative characterization of varieties of groups (and not only of groups, but of algebraic systems in general): a class of groups is a variety if and only if it is closed under the operation of forming epimorphs of subgroups of cartesian products; or, by a remark of Kogalovskil, if and only if it is closed under the operation of forming epimorphs of subcartesian products. In this context the following question, which I owe to Evelyn Nelson, is of interest: ${ }^{2}$

Problem 3. Let $\mathfrak{X}$ be a class of groups, and $\mathfrak{Y}$ the class of subgroups of epimorphs of cartesian products of subgroups of members of $\mathfrak{X}$. Is $\mathfrak{Y}$ necessarily a variety?

So far all we have dealt with is not peculiar to groups, but belongs properly to universal algebra. The universal algebra aspects of the theory have been described in an address given by Alfred Tarski to the Colloquium on Logic and Foundations of Mathematics, Hannover 1966; and Professor Tarski has kindly made a summary of his address,

2 [Note added February 1967.] In the actual lecture I misstated the problem. Professor Gratzer subsequently drew my attention to the (positive) solution of this problem implicit in the results announced by Don Pigozzi [9]. 
to be published in the Proceedings of that conference, available to me, and has thereby drawn my attention to some recent, mostly unpublished work.

We turn now to a more properly group-theoretical notion: if $\mathfrak{X}$ and $\mathfrak{Y}$ are classes of groups, we introduce the product $\mathfrak{X Y}$ as the class of groups that are extensions of groups in $\mathfrak{X}$ by groups in $\mathfrak{Y}$, that is groups $G$ with a normal subgroup $N \in \mathfrak{X}$ such that $G / N \in \mathfrak{Y}$. This multiplication of classes of groups is neither commutative nor associative, not even power-associative: if $\mathfrak{X}$ is the class of cyclic groups, then $\mathfrak{X}(\mathfrak{X} X)$ is properly contained in $(\mathfrak{X} X) \mathfrak{X}$. For varieties of groups, however, this multiplication has two important features: the products of two varieties is again a variety, and multiplication is associative (Hanna Neumann, 1956). Thus the set of varieties has not only the structure of a complete lattice, but also that of a semigroup. Moreover, multiplication respects the lattice order. The semigroup has a zero, namely the variety $\mathfrak{D}$ of all groups, and a unit element, namely the variety $\mathbb{E}$ of trivial groups, and apart from these two extreme elements it turns out to be a free semigroup, freely generated by the so-called indecomposable varieties (B. H. Neumann, Hanna Neumann, and Peter M. Neumann, 1962; A. L. Šmel'kin, 1962): thus every variety other than $\mathfrak{D}$, $\mathbb{E}$ has a unique factorization, unique even with respect to the order in which the factors occur, into indecomposable varieties. Many varieties are known to be indecomposable (Peter M. Neumann, 1963), but the question just how many indecomposable varieties of groups there are is equivalent to Problem 1.

3. Laws. We turn to the laws that determine a variety. They are of the form

$$
u\left(x_{1}, x_{2}, \cdots, x_{n}\right)=v\left(x_{1}, x_{2}, \cdots, x_{n}\right)
$$

where $u, v$ are words; or, as we are dealing with groups, we can put $v^{-1} u=w$ and write our laws in the form

$$
w\left(x_{1}, x_{2}, \cdots, x_{n}\right)=1 .
$$

If a variety is determined by finitely many laws, then a single law will do: we just multiply all the left-hand sides together, after changing the numbering of the variables so that different laws involve different variables. Are all varieties of groups definable by a single law? This is equivalent to the following open question.

PROBLem 2'. Is there a variety of groups that can not be defined by a finite set of laws?

This is, in fact, no new problem, but only a different formulation of our Problem 2. For if a variety $\mathfrak{B}$ requires an infinite set of laws, say 
$w_{1}=w_{2}=w_{3}=\cdots=1$, and no finite subset suffices to define $\mathfrak{B}$, then the sequence of varieties

$$
\mathfrak{B}_{1} \supseteq \mathfrak{B}_{2} \supseteq \mathfrak{B}_{3} \supseteq \cdots \supseteq \mathfrak{B},
$$

where $\mathfrak{B}_{k}$ is defined by the first $k$ laws, $w_{1}=w_{2}=\ldots=w_{k}=1$, must descend properly infinitely many times; and the converse is equally straightforward.

Again this question can be asked for other algebraic systems than groups. R. C. Lyndon [6] has made an example of a finite algebraic system, of order 7 , whose laws do not follow from any finite set among them. The same can be done with a groupoid of order 3 , according to V. L. Murskir [7], and even with a semigroup of order 6, according to Peter Perkins [8]. Recently A. K. Austin [3] has exhibited a variety of semigroups that requires infinitely many laws to define it; see also A. P. Biryukov [4]. ${ }^{3}$

Problem 2 ' is known as the "finite basis" problem, which is a misnomer: a "basis" in common mathematical parlance is a generating set with appropriate independence or irredundancy properties. Thus the finite basis problem would properly be the following.

Problem 4. Is there an infinite irredundant set of group laws, that is an infinite set of laws such that the variety it defines is distinct from each of the varieties obtained by omitting one of the lawes?

If this is answered in the affirmative, it immediately also gives the answer " $2 \mathbb{N}_{0}$ " to Problem 1 and thus answers Problem 2 negatively and Problem $2{ }^{\prime}$ positively. This is, in fact, the way in which the corresponding problems for groupoids and loops were solved (vide supra).

Dr. L. G. Kovács informs me that he can prove that if there is a locally finite variety that does not satisfy the descending chain condition for subvarieties, then it has $2^{N_{0}}$ subvarieties-and he has some more general results.

Though these problems are still the most baffling and important problems for groups in general, interesting progress has been made in a number of special cases. In 1952 R. C. Lyndon showed that the laws of every nilpotent variety are finitely based (a variety is nilpotent if it consists of nilpotent groups; and so with other properties). In the same year Graham Higman showed that certain natural ways of making new laws from other laws will only lead to finitely based varieties. In 1959 Graham Higman extended R. C. Lyndon's result to product varieties $\mathfrak{U} \mathfrak{B}$, where $\mathfrak{U}$ is nilpotent and $\mathfrak{B}$ finitely based. Then D. C. Cross, M. B. Powell, Sheila Oates, and finally Sheila Oates and

\footnotetext{
'I owe this reference to Professor Gratzer.
} 
M. B. Powell jointly, all working with Graham Higman at Oxford, made a successful attack on the basis problem for the laws of a finite group, proving in 1963 that all the laws that hold in a given finite group follow from a finite set among them. This important result, proved by difficult and delicate methods, has now been improved, and the proof simplified by L. G. Kovács and M. F. Newman. Contrast with this Lyndon's 1954 example (vide supra). Recently D. E. Cohen has used the methods of Graham Higman (1952) to prove that the laws of a metabelian variety of groups are finitely based.

Now let us look at a different kind of question: what can be said about the variety when the laws are given? The oldest and most famous problem here is due to W. Burnside:

Problem 5. Is the Burnside variety $\mathfrak{B}_{e}$, of groups of exponent $e$, locally finite?

This is in fact an infinite sequence of problems, one for each exponent $e$. The answer is known to be positive for $e=2,3,4,6$, and a negative solution has been announced by S. I. Adyan and P. S. Novikov at the ICM Moscow in August, 1966, for large odd $e$ (an earlier announcement by P. S. Novikov was premature); and at the same meeting J. L. Britton produced evidence pointing the same way. There are, however, still plenty of finite exponents left for which the problem is open.

When the answer to the Burnside problem is negative or open, the Restricted Burnside problem becomes interesting. This asks whether among the finite groups in $\mathfrak{B}_{e}$ with $d$ generators there is a maximal one. To this question L. G. Kovács (1967) has recently given an intriguing new formulation:

Problem 6. Do the locally finite groups in $\mathfrak{B}_{e}$ form a variety?

The equivalence of this with the Restricted Burnside problem is not trivial. A well-known result of A. I. Kostrikin (1959) gives a positive answer for $e=p$, a prime.

Instead of trying to make the Burnside problem more manageable, one can also try to make it harder. To do this, we need some more definitions and notation.

4. Verbal subgroups. We now look not just at sets of laws that define a variety, but at all the laws that hold in the variety. Thus we take a countably infinite alphabet $x_{1}, x_{2}, x_{3}, \ldots$ and ask for all the words $v$ in these variables that are laws in the variety $\mathfrak{B}$; we think of the words as elements of the free group $X_{\infty}$ generated by $\left\{x_{1}, x_{2}, x_{3}, \cdots\right\}$. Then the set $V$ of laws of $\mathfrak{B}$ consists just of those $v=v\left(x_{1}, \cdots, x_{n}\right)$ that take the value 1 whenever elements $a_{1}, \cdots, a_{n}$ 
of a group $A \in \mathfrak{B}$ are substituted for $x_{1}, \cdots, x_{n}$. Then $V$ is a subgroup of $X_{\infty}$, and in particular a fully invariant subgroup. Denote by $V(A)$ the subgroup of $A$ generated by all values of words $v \in V$ on substitution of elements of $A$ for the variables. Then $V(A)$ is a verbal subgroup of $A$, and the connection between $V$ and the variety $\mathfrak{B}$ is that

$$
A \in \mathfrak{B} \text { if and only if } V(A)=\{1\} .
$$

Verbal subgroups are fully invariant, and in free groups the converse is also true. As $\mathfrak{B}$ and $V$ are in precise correspondence, we have a one-to-one correspondence between the lattice of varieties of groups and the lattice of verbal subgroups, and this is a lattice anti-isomorphism. Thus all the questions about the lattice of varieties can be translated into questions about the lattice of verbal subgroups of the free group $X_{\infty}$.

Now put $X_{d}=\operatorname{gp}\left(x_{1}, x_{2}, \cdots, x_{d}\right) \leqq X_{\infty}$; this is a free group of rank $d$. If we form $X_{d} \cap V$, we obtain a fully invariant, hence verbal subgroup of $X_{d}$; this consists of the laws in $d$ variables of the variety $\mathfrak{B}$. In this way we map the lattice of verbal subgroups of $X_{\infty}$ on to that of $X_{d}$-it is easy to see that every verbal subgroup of $X_{d}$ is the intersection of $X_{d}$ with the verbal subgroup of $X_{\infty}$ it generates. This is a lattice homomorphism, and the equivalence classes defined by its kernel congruence are intervals, say $V^{(d)} \leqq V \leqq V_{(d)}$, and correspondingly for the varieties: $\mathfrak{B}_{(d)} \subseteq \mathfrak{B} \subseteq \mathfrak{B}^{(d)}$. Here $\mathfrak{B}_{(d)}$ and $\mathfrak{B}^{(d)}$ are the smallest and largest varieties, respectively, that have the same laws in $d$ variables as $\mathfrak{B}$. They can be easily and independently described: $\mathfrak{B}_{(d)}$ is the variety generated by all $d$-generator groups in $\mathfrak{B}$, and $\mathfrak{B}^{(d)}$ is the variety defined by all $d$-variable laws of $\mathfrak{B}$; or, equivalently, $\mathfrak{B}^{(d)}$ consists of those groups whose $d$-generator subgroups are in $\mathfrak{B}$. Now one can translate the various problems about the lattice of varieties or the lattice of verbal subgroups of $X_{\infty}$ to problems about the lattice of verbal subgroups of $X_{d}$ : however, excepting the trivial cases $d=0$ and $d=1$, where the problems vanish, no really new problems arise, as the lattice of verbal subgroups of, for example, $X_{2}$ contains an isomorphic copy, indeed many isomorphic copies, of the lattice of verbal subgroups of $X_{\infty}$. So for new problems we have to look in other directions.

Problem 7. Let $\mathfrak{B}$ be a locally finite variety and $d \geqq 1$ an integer. Is $\mathfrak{B}^{(d)}$ locally finite?

This can be called the Extended Burnside problem, as the classical Burnside problem is the special case $d=1$ of this. It is, of course, again an infinite set of problems, one for each pair $(\mathfrak{B}, d)$. As we know, 
modulo Adyan, Novikov, Britton, that the answer is negative in some cases, we go on to ask:

PROBLEM 8. Is there, to each locally finite variety $\mathfrak{B}$, an integer $d=d(\mathfrak{B})$ such that $\mathfrak{B}^{(d)}$ is locally finite?

Again, when the answer to Problem 7 is negative for a particular pair $(\mathfrak{B}, d)$, we can formulate a Restricted Extended Burnside problem, using again the idea of L. G. Kovács as with Problem 6:

PRoBlem 9. Do the locally finite groups in $\mathfrak{B}^{(d)}$, where $\mathfrak{B}$ is a locally finite variety, form a variety?

Moreover, local finiteness is only one of the properties that can be considered in this way - there are many others, but there is no need to state the corresponding problems explicitly.

We have already begun to vary the number $d$ of variables or generators. If we let $d$ range over all positive integers, we get to each variety $\mathfrak{B}$ two sequences that converge to it:

$$
\mathfrak{B}_{(1)} \subseteq \mathfrak{B}_{(2)} \subseteq \mathfrak{B}_{(3)} \subseteq \cdots \subseteq \mathfrak{V} \subseteq \cdots \subseteq \mathfrak{B}^{(3)} \subseteq \mathfrak{B}^{(2)} \subseteq \mathfrak{B}^{(1)},
$$

with

$$
\bigvee_{d} \mathfrak{B}_{(d)}=\mathfrak{B}=\bigwedge_{d} \mathfrak{B}^{(d)}
$$

(this is due to Hanna Neumann, 1956). Examples are known where the sequence on the left ascends properly at every step; and M. F. Newman has found an example of a variety where the sequence on the left ascends properly for a few steps, then remains stationary for one step, and then ascends properly again for the remaining infinitely many steps. It is not known whether the sequence on the right can go on descending properly - of course not: if an example were known, it would at once provide a negative answer to Problem 2. But the converse implication is not true, or at any rate not evident, and we accordingly have to ask:

PROBLEM 10. Is there to each variety $\mathfrak{B}$ an integer $d=d(\mathfrak{B})$ such that $\mathfrak{B}=\mathfrak{B}^{(d)}$ ?

Differently put: do all the laws of a variety follow from those in a certain finite number of variables, the number, of course, depending on the variety? As we have already remarked, a positive answer to Problem 2 would entail a positive answer to Problem 10; and clearly a positive answer to Problem 10 would entail a positive answer to Problem 8 ; but not conversely.

The verbal subgroups $V$ of $X_{\infty}$ are, inter alia, characteristic subgroups of $X_{\infty}$, and as an aside I should like to draw attention to another tantalizing problem: 
Problem 11. Is every characteristic subgroup of the free group $X_{\infty}$ of (countably) infinite rank fully invariant, hence verbal in $X_{\infty}$ ?

5. Free groups. Let us now turn to the factor groups of the verbal subgroup. If $\mathfrak{B}$ is a variety and $V$ the corresponding verbal subgroup of $X_{\infty}$, consisting of the words that are identically 1 in the groups in $\mathfrak{B}$, we put

$$
F_{\infty}(\mathfrak{B}) \cong X_{\infty} / V, \quad F_{d}(\mathfrak{B}) \cong X_{d} /\left(X_{d} \cap V\right),
$$

and call these the $\mathfrak{B}$-free groups of rank $\boldsymbol{\aleph}_{0}$ or $d$, respectively-the corresponding groups for uncountably infinite rank are similarly defined, but need not concern us. They are characterized by having a set of generators called a "free basis", namely the images under the canonic epimorphism of $\left\{x_{1}, x_{2}, x_{3}, \cdots\right\}$ or $\left\{x_{1}, x_{2}, \cdots, x_{d}\right\}$, respectively, with the property that every mapping of the set in to a group $A$ of the variety can be extended to a homomorphism of the free group into $A$.

The $\mathfrak{D}$-free groups $F_{\infty}(\mathfrak{D}) \cong X_{\infty}, F_{d}(\mathfrak{D}) \cong X_{d}$ are also called "absolutely free", other $\mathfrak{B}$-free groups are called "reduced free" or "relatively free". Except in the trivial case of \&-free groups, the rank is uniquely determined by the $\mathfrak{B}$-free group, namely as the minimal cardinal number of a generating set. A free basis always has this minimal cardinality; and in all known cases a converse is also true when the rank is finite. Thus for example every generating set of $F_{d}(\mathcal{D})$ that consists of $d$ elements is a free basis.

PROBLEM 12. Is every generating set of $F_{d}(\mathfrak{B})$ consisting of $d$ elements a free basis, where $\mathfrak{B}$ is an arbitrary variety and $d$ a positive integer?

Let me remind you that a group $A$ is called a Hopf group if it is not isomorphic to any proper factor group of itself, or, equivalently, if every ependomorphism of $A$ is an automorphism. The original question of Heinz Hopf whether finitely generated groups are Hopf groups was first answered positively for absolutely free groups, and then for reduced free groups of some other varieties. As finitely generated nonHopf groups are known to exist, it makes sense to ask if there are reduced free groups among them:

PROBLEM 12'. Is every reduced free group of finite rank a Hopf group? This problem is, however, equivalent to its predecessor, in fact is only a reformulation of it.

In this context I should like to mention an intriguing conjecture of Peter M. Neumann:

CONJECTURE. If $\mathfrak{B}$ is a variety and $d$ a positive integer, and if $\mathfrak{B}$ con- 
tains $2^{\aleph_{0}}$ nonisomorphic d-generator groups, then $\mathfrak{B}$ also contains a $d$-generator non-Hopf group; and conversely.

Of the numerous important properties of absolutely free groups I will mention only two: every subgroup of an absolutely free group is absolutely free-this is known as the Schreier property; and every absolutely free group of rank greater than 1 contains free groups of arbitrary finite rank and of rank $\aleph_{0}$. It is easy to see that the free groups not only of $\mathfrak{D}$ but also of $\mathfrak{A}$ and of $\mathfrak{A}_{p}$ with prime exponent $p$ have the Schreier property; and it has been proved by Peter M. Neumann and James Wiegold, and by an improved argument by Peter M. Neumann and M. F. Newman, that these are indeed all the varieties whose free groups have the Schreier property.

On the other hand, no variety $\mathfrak{B}$ other than $\mathfrak{D}$ and $\mathbb{E}$ is known in which $F_{d+1}(\mathfrak{B})$ can be embedded in $F_{d}(\mathfrak{B})$, for any finite rank $d$. A variety $\mathfrak{B}$ is called "regular" if it has this property, that is if none of its free groups $F_{d}(\mathfrak{B})$ of finite rank contains a group isomorphic to $F_{d+1}(\mathfrak{B})$.

Problem 13. Are all varieties $\neq \mathfrak{D}$, $\&$ regular?

Auslander and Lyndon proved that if $R$ and $S$ are normal subgroups of a free group $F$ and if their derived groups are comparable, say $S^{\prime} \leqq R^{\prime}$, the $S \leqq R$. This has been generalized by various authors, the most recent result being that for arbitrary nontrivial verbal subgroups $V \subseteq X_{\infty}, V(S) \leqq V(R)$ implies $S \leqq R$ - this has been announced by M.A. Bron'stein.

6. Categories. We consider the category $\mathfrak{C}(\mathfrak{B})$ of all homomorphisms of groups in $\mathfrak{B}$ into groups in $\mathfrak{B}$. Here the $\mathfrak{B}$-free groups are projective objects: if $F$ is $\mathfrak{B}$-free and $A$ and $B$ are in $\mathfrak{B}$, then to every epic $\alpha$

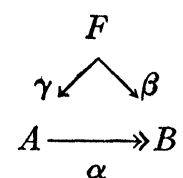

and every $\beta$ there is a $\gamma$ to make this diagram commutative. However, varieties $\mathfrak{B}$ are known where $\mathfrak{C}(\mathfrak{B})$ contains projective objects that are not $\mathfrak{B}$-free; all the known ones are of finite exponent divisible by at least two distinct primes (P. Hall, 1954).

PRoBlem 14. For what varieties $\mathfrak{B}$ are the projective objects of $\mathfrak{C}(\mathfrak{B})$ always $\mathfrak{B}$-free?

Another question that has recently arisen in a different context is the following: 
Problem 15. What varieties $\mathfrak{C}(\mathfrak{B})$ have the property that every epic monic is invertible, that is to say, an isomorphism?

It is known that this is the case in all those varieties $\mathfrak{B}$ with the embedding property: every amalgam of two groups in $\mathfrak{B}$ can be embedded in a group in $\mathfrak{B}$; but the only known varieties with the embedding property are $\mathfrak{D}, \mathfrak{A}$, and $\mathfrak{A}_{e}$ for arbitrary exponent $e$. If there is another, it must be nonabelian, but all its finite groups must be abelian. Thus we are led to ask:

PROBLEM 16. Is there a variety that contains nonabelian infinite groups but no nonabelian finite groups?

If $\mathfrak{B}$ is the variety generated by the icosahedral group (any other finite nonabelian simple group will serve as well), then $\mathfrak{C}(\mathfrak{B})$ has noninvertible epic monics; but at least all epic monic endomorphisms are invertible in this category. Is this a general feature?

PROBLEM 17. Is there a variety $\mathfrak{B}$ such that $\mathfrak{e}(\mathfrak{B})$ contains an epic monic endomorphism that is not invertible?

[Added in proof, July 10, 1967.] Dr. Peter M. Neumann has kindly drawn my attention to the fact that $\$ 6$ above is full of errors and omissions. He has also constructed an example that solves Problem 17 positively. See his forthcoming paper Splitting groups and projectives in varieties of groups, Quart. J. Math. Oxford (2) 19 (1968).

7. Quasi-varieties. I really still owe you an explanation of the statement I made at the beginning, namely that the class $\mathfrak{D}$ of all groups is a variety. Groups used to be defined in terms of a binary multiplication, and the axioms consisted partly of laws, such as the associative law, partly of certain existence or unicity postulates. However, if groups are instead defined in terms of a binary multiplication and a unary inversion, one can make the axioms equational. Indeed it can be done by means of a single law.

A number of authors have defined groups, or abelian groups, not by a single law, but by a single identical implication:

$$
\begin{aligned}
& \text { if } u\left(x_{1}, x_{2}, \cdots, x_{n}\right)=v\left(x_{1}, x_{2}, \cdots, x_{n}\right), \\
& \text { then } u^{\prime}\left(x_{1}, x_{2}, \cdots, x_{n}\right)=v^{\prime}\left(x_{1}, x_{2}, \cdots, x_{n}\right) .
\end{aligned}
$$

Classes of algebras defined by identical implications have been called "quasi-varieties". Every variety is obviously a quasi-variety, but not conversely. This leads me to a final question, that I shall have to leave quite vague:

PROBLEM 18. What formal properties of an identical implication, or a set of identical implications, will ensure that the quasi-variety it defines is $a$ variety? 


\section{REFERENCES}

Full references will be found in:

1. Hanna Neumann, Varieties of groups, Ergebnisse der Mathematik und ihrer Grenzgebiete, Band 37, Springer-Verlag, Berlin, 1967.

2. - Varieties of groups, Proc. Internat. Conf. Theory of Groups, Austral. Nat. Univ. Canberra, August 1965, p. 251-259, Gordon and Breach, New York, 1967. Additional references:

3. A. K. Austin, A closed set of laws which is not generated by a finite set of laws, Quart. J. Math. Oxford (2) 17 (1966), 11-13.

4. A. P. Biryukov, On infinite families of identities in semigroups, Algebra i Logika Seminar 4 (1965), pp. 31-32. (Russian)

5. Trevor Evans and B. H. Neumann, On varieties of groupoids and loops, J. London Math. Soc. 28 (1953), 342-350.

6. R. C. Lyndon, Identities in finite algebras, Proc. Amer. Math. Soc. 5 (1954), 8-9.

7. V. L. Murskii, 1966, unpublished (quoted from Tarski, 1967).

8. Peter Perkins, Ph.D. thesis, University of California, Berkeley, 1966 (quoted from Tarski, 1967).

9. Don Pigozzi, On some operations on classes of algebras, Notices Amer. Math. Soc. 13 (1966), Abstract 639-1, p. 829.

10. Alfred Tarski, Equational theories, Proc. Colloquium on Logic and Foundations of Mathematics, Hannover 1966, 1967 (to appear).

The University of Wisconsin AND

The Australian National University 